\title{
Analysis of The Electrodermal Activity Signals for Different Stressors Using Empirical Mode Decomposition
}

\author{
${ }^{* 1}$ Ramis İleri, ${ }^{1}$ Fatma Latifoğlu \\ ${ }^{1}$ Erciyes University,Department of Biomedical Engineering,Kayseri/Turkey, ramissileri@ gmail.com, \\ ${ }^{1}$ Erciyes University,Department of Biomedical Engineering,Kayseri/Turkey, flatifoglu@erciyes.edu.tr, \\ Research Paper \\ Arrival Date: 03.08.2019 \\ Accepted Date: 10.05.2020
}

\begin{abstract}
In this study, Electrodermal Activity (EDA) signals were analyzed to evaluate the changes between physical stress, cognitive stress, and emotional stress. For this purpose, energy and variance properties of the EDA signals in the time domain were analyzed for each case and as short-time frames. In addition, the EDA signals were decomposed using the Empirical Mode Decomposition (EMD) method, and the sub-band signals were analyzed for each case. Further, the Short Time Fourier Transform (STFT) method was used to analyze the in the time-frequency domain of these signals. Also, according to obtained features, EDA signals were classified to determine the stages. Simulated results show that, the EDA and subband EDA signals were found to be significantly different in terms of cognitive stress $(p<0.05)$. Also, the features obtained from the EMD subbands were classified using Support Vector Machines (SVM), K-Nearest Neighbors (KNN), and Multi-Layer Perceptron (MLP) methods for different situations and classifier performances were compared. In the classification of cognitive stress period and first rest period, the best classification performance was achieved as $97.36 \%, 84,21 \%$, and 81,57\% using MLP, SVM and KNN classifier respectively compared to other situations.
\end{abstract}

Keywords: Electrodermal Activity (EDA), Empirical Mode Decomposition (EMD), Short Time Fourier Transform (STFT), Artificial Neural Network (ANN)

\section{INTRODUCTION}

Electro Dermal Activity (EDA) is defined as the electrical activity that is recorded by the sweat glands and electrodes, which are formed from adjacent epidermal and dermal layers and placed on special areas on the skin surface [1,2]. They are recorded as conductivity changes from the skin surface. These recorded signals are called electrodermal activity signals. Obtained, these signals are bio signals used to measure the change in skin resistance caused by an increase in eccrine sweat gland activity [3, 4]. An EDA is a way of obtaining the activity of sweat glands attached to the autonomic nervous system, as a parameter. An EDA is a simple, noninvasive and reproducible electrophysiological technique that is useful for investigating sympathetic nervous system function. Physically, an EDA is the change in the response of different types of stimuli to the electrical properties of the skin [5]. Small changes in the electrical properties of the skin are measured with the help of finger sensors and electrodes. Electrodermal activity is associated with stress and relaxation [6]. The electrical resistance of the skin changes rapidly during the mental, physical, and emotional arousal. EDA, which is robust, cost-effective, and non-invasive is used to detect cognitive, emotional, and motor processes [7, 8]. Fluctuations in skin conductance are linked to a specific set of brain circuitry [9].

In literature, there are some studies analyzing EDA in mental, physical, and emotional states, and for different diseases. Cornelia at al.[10] developed a personal health system to detect stress. For this purpose, a group of 33 people underwent psychosocial stress caused by mental stress and social-evaluative threat resulting from the resolution of arithmetic problems under time pressure in the office environment. During this process, EDA signals were recorded from the subjects. When the data was analyzed, the EDA showed that the distribution of peak height and instant peak velocity conveyed information about a subject's stress level. A maximum accuracy of $82.8 \%$ was achieved for discriminating stress from cognitive load. Rabavilas A.D. examined the relationship between alexithymia and arousal level in patients with anxiety states. The results showed that patients with high alexithymia had significantly higher levels of electrodermal stimulation and slower recovery times compared to lower alexithymia patients [11].

In another study, nonspecific EDAs and specific EDAs were examined in two experimental sessions with two groups including 12 hyperactive children and 12 normal controls. In 
hyperactive children, lower basal skin conductivity, less specific EDA, and smaller specific EDAs were found to be lower than in normal children. The stimulatory drugs administered to a group of hyperkinetic children were found to be prone to increase basal level conductivity and nonspecific GSR activity toward normal child levels [12]. Healey J. A. and Picard R. W. provides methods for collecting and analyzing physiological data during realworld driving tasks to determine the relative stress level of drivers. Electrocardiogram, electromyogram, skin conductivity, and respiration were recorded continuously while drivers followed a route on open roads in the Boston area. At the end of the study, most of the drivers showed that skin conductivity and heart rate measurements were the most closely related to the level of drive stress [13]. In literature studies, EDA signals have been analyzed according to amplitude of the time response of the signals. The main purpose of this study is to evaluate whether there is a difference between different stress situations by using the features obtained from EDA signals without EMD using and the properties obtained by using EMD. Another aim of this study is to evaluate which type of stress is identified better using the features obtained from the subband (IMF) signals decomposed by EMD method. For this aim we analyzed EDA signals in time and frequency domain using STFT and EMD methods. EDA signals recorded during different stages including physical stress, cognitive stress, and emotional stress from healthy subjects were analyzed using EMD method. To the best of our knowledge, this study is the first study in the literature analyzing and classifying changes in EDA signals in different stress situations using the EMD method. This paper is organized as follows. Section 2 provides Materials and Methods. The results are discussed and explained in Section 3, including an analysis of EDA signals. Finally, in Section 4, the paper is concluded.

The applied process in this study is seen from Figure1.

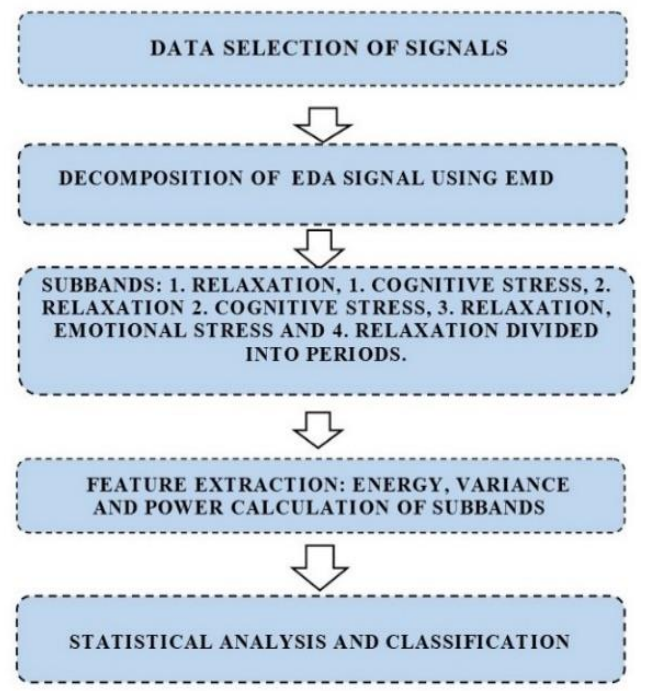

Figure 1. Steps followed in the study

\section{MATERIALS AND METHODS}

In this study, for the analysis of EDA signals, the process steps are described in below flowchart.

\subsection{Participants and Data Acquisition}

In this study, data was obtained from the 'physio.net' database. In this database SPO2 (atrial oxygen saturation), HR (heart rate), body temperature, and EDA signals are available and recorded from 19 healthy university students. Only EDA signals were used for analysis in this study [14, 15]. The sampling frequency of EDA signals is $8 \mathrm{~Hz}$.

Signals were recorded in seven stages as first relaxation, physical stress, second relaxation, cognitive stress, third relaxation, emotional stress, and forth relaxation period. A certain procedure was applied for the signals recorded from subjects at each stage as seen in Figure 2.

1-First Relaxation: Five minutes rest.

2-Physical Stress: Stand for one minute, walk on a treadmill at one mile per hour for two minutes, then walk/jog on the treadmill at three miles per hour for two minutes.

3-Second Relaxation: Five minutes rest.

4-Cognitive Stress: Count backwards by sevens, beginning at 2485 for three minutes. The Stroop test consisted of regarding the names of colors written in a different color ink then saying what color the ink was. In both tests, the volunteers were alerted to errors by a buzzer.

5-Third Relaxation: Five minutes rest.

6-Emotional Stress: The volunteer was told he/she would be shown a five-minute clip from a horror movie after minute. After the minute of anticipation, a clip from a zombie apocalypse movie, The Horde was shown.

7-Forth Relaxation : Five minutes rest.[14].

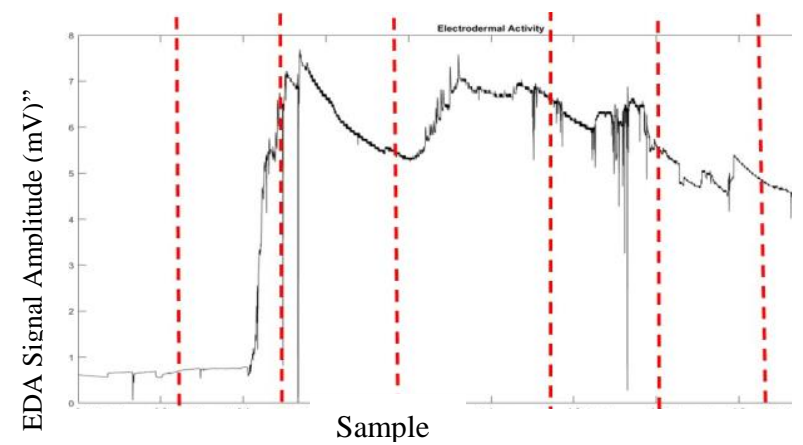

Figure 2. Decomposition of signals into seven sections [14].

At this stage of the study, physical, cognitive, and emotional stress was decomposed into sub bands by applying EMD to analyze the EDA signals according to sub bands. The energy, power, and frequency features of these sub band signals were calculated. The STFT method was also used for frequency analysis of the EDA signals. 


\subsection{Empirical Mode Decomposition}

Empirical Mode Decomposition method proposed by Huang decomposes any given data into intrinsic mode functions (IMF) and residual signal using shifting process [16]. EMD is an adaptive technique that allows decomposition of nonlinear and non-stationary data into intrinsic mode functions (IMF)[17].

EMD method has been used in the literature to decompose physiological signals such as EEG and ECG and to remove noise from the signals. Slimane at al.[18] used the EMD method to detect the QRS complex from ECG signals. Valesko at al.[19] used the EMD method to remove noise from ECG signals. Bajaj at al.[20] proposed a method for the diagnosis of Epilepsy disease using the EMD method to EEG signals. Zhang at al.[21] proposed an EMD method based algorithm to remove noise from EEG signals. In addition, Gautam at.[22] used the EMD method for noise elimination of EDA signals.

In this study EDA signals were decomposed into 5 sub band signals by using EMD method. Here, we tried different decomposition levels and the 5 level decomposition process was sufficient to reveal the inter-state variation[16, 23-25]. Figure 3 shows the decomposed signals into $5 \mathrm{IMF}$ and residual component using the EMD method.

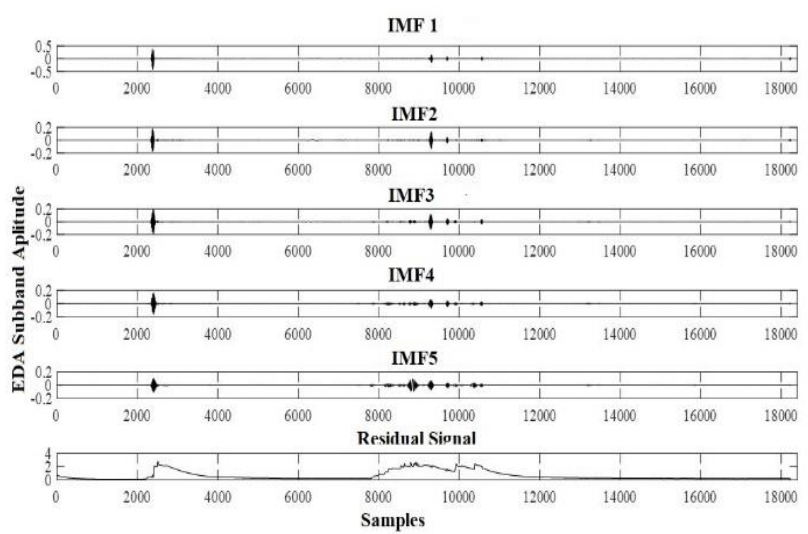

Figure 3. EDA signals divided into five sub bands by the EMD method

\subsection{Frequency Analysis of the EDA Signals}

The STFT method was used to show the time and frequency components and to evaluate visually of EDA signals. The STFT method is proposed by Dennis Gabor (1946) adapted the Fourier transform to analyze only a small section of the signal at a time. This technique is called windowing the signal. The STFT method maps a signal into a twodimensional function of time and frequency. Therefore, the STFT provides the time- and frequency-based views of a signal. The STFT method uses the fallowing equation;

$\operatorname{STFT}(t, w)=\int_{-\infty}^{t} f(\tau) \vartheta(\tau-t) e^{-j w \tau} d \tau$
Where $\vartheta(\tau-t)$ is defined as window function. In this study, STFT method was used to visually show both time and frequency information of EDA signals and IMF signals. Figure 4 shows the STFT sonogram image of raw EDA signals.

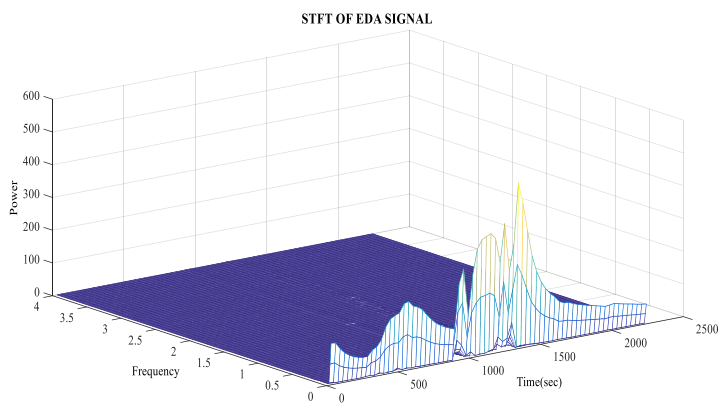

Figure 4. STFT of Raw EDA Signal

\subsection{Calculated Features from EDA Signals}

To analysis EDA signals in time and frequency domain, we calculated energy and variance of EDA signals in time domain. Also power spectral density (PSD) of EDA signals were obtained using Welch method and power feature from PSD graphics were calculated for each state. Also, we analyzed sub band signals according to variance and energy terms.

\subsection{Classification of EDA signals for different situations}

At this stage of study, Artificial Neural Networks (ANNs), Support Vector Machines (SVM), K Nearest Neighbors (KNN) methods were applied to EDA signals for classification of each stage using obtained features. Also, classification performance of the used methods was compared. Energy and variance values of sub band signals were used for classification of relaxation, cognitive stress, physical stress and emotional stress stages. Classification process was performed for first rest period and physical stress period, first rest period and cognitive stress period, and first rest period and emotional stress period.

ANN is a mathematical model inspired by the information processing methods of biological nervous systems such as the brain. The ANN architecture of interconnected neurons is structured for specific applications such as data classification [26]. During classification of EDA signals with ANN, Multilayer Perceptron (MLP) method is used. Two hidden layers were used for network model to get best classification performance and $\mathrm{k}$ value was selected as 5 for $\mathrm{k}$-fold cross validation.

KNN (K-Nearest Neighbors) is one of algorithm among many algorithms used in data mining and machine learning. It is a classifier algorithm where the learning is based "how similar" a data (a vector) from other [22]. Among the various classification methods, Support Vector Machines (SVMs) have emerged as a powerful classification technique. SVM 
solves a convex optimization problem which leads to a globally optimal solution [23]. The detailed information about ANN , KNN and SVMs exists in ref [27-29]. All classification process was performed using MATLAB Classification Learner Toolbox.

\subsection{Statistical Analysis}

We tested the data for normality and found that the data did not have normal distribution. Therefore, we performed the Mann Whitney U-Test on our data. The Mann-Whitney U test is a non-parametric test that can be used in place of an unpaired t-test. It is a nonparametric test that is used to compare two population means that come from the same population, it is also used to test whether two population means are equal or not.

\section{RESULTS}

The time and frequency analysis of the EDA signals were obtained by using the STFT method and the obtained timefrequency sonogram is shown in Figure 4. Also, the energy and the variance of the EDA signal has been calculated for all situations.

\subsection{Energy and Variance Features of EDA signals}

In order to determine whether there is a statistically significant difference between the different states of EDA signals, energy and variance variables were analyzed statistically. In each stage of EDA signals variance and energy features are calculated individually and compared according to first relaxation stage. The $p$ values obtained are given in Table 1.

When the raw EDA signals are evaluated in terms of energy properties, only a significant difference was found between first relaxation and cognitive stress. When evaluated in terms of variance feature, there was a significant difference between first relaxation in both cognitive stress and physical stress situations.

Table 1. Mann Whitney U-Test Result

\begin{tabular}{lccc}
\hline \hline & & $\begin{array}{c}\text { ENERGY } \\
\text { Sig(2-tailed) }\end{array}$ & $\begin{array}{c}\text { VARIANCE } \\
\text { Sig(2-tailed) }\end{array}$ \\
\cline { 3 - 4 } $\begin{array}{l}\text { First Relaxation } \\
\text { Physical Stress }\end{array}$ & $\rightarrow$ & 0,082 & $0,04 *$ \\
$\begin{array}{l}\text { First Relaxation } \\
\text { Cognitive Stress }\end{array}$ & $\rightarrow$ & $\mathbf{0 , 0 0 *}$ & $\mathbf{0 , 0 0 *}$ \\
$\begin{array}{l}\text { First Relaxation } \\
\text { Emotional Stress }\end{array}$ & $\rightarrow$ & 0,052 & 0,125 \\
\hline \hline
\end{tabular}

\section{Analysis of EDA signals using EMD}

EDA signals are decomposed into five sub-bands using the EMD method. The sub bands are divided into seven stages: first rest, physical stress, second rest, cognitive stress, third rest, emotional stress, and fourth rest, considering the determined signal lengths. Energy and variance values of each stage for the five sub bands signals were calculated. Also, power and energy calculation of the EDA signals for each mode was performed from PSD graphics. The power of the signals which are windowed 10 second frames $(10 * 8=$ 80 samples) in frequency domain was calculated.
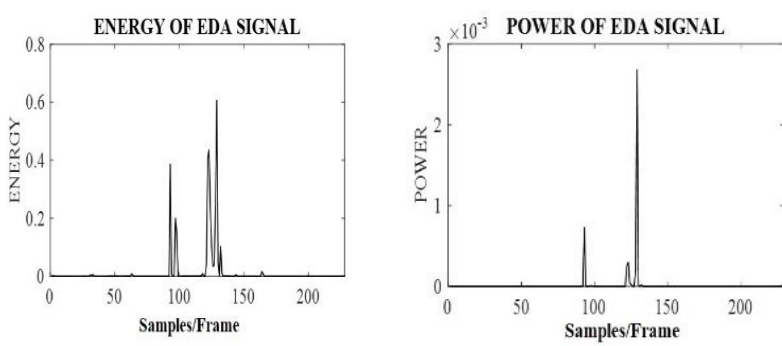

Figure 5.a Energy of IMF2 Subband of EDA Signal Figure 5.b Power of IMF2 Subband of EDA Signal

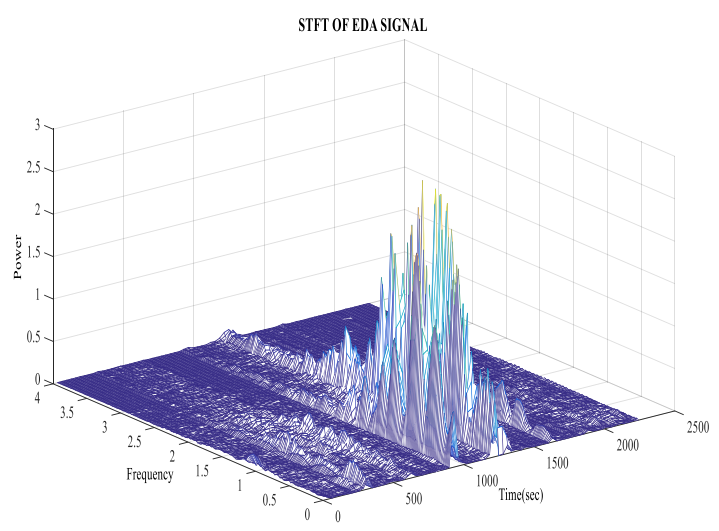

Figure 6. STFT sonogram of IMF2 EMD Subband Signal 
Table 2. Statistical Analysis Results

\begin{tabular}{|c|c|c|c|c|c|c|}
\hline ENERGY & & $\begin{array}{c}\text { SUBBAND } 1 \\
\text { Sig(2-tailed) }\end{array}$ & $\begin{array}{c}\text { SUBBAND } 2 \\
\text { Sig(2-tailed) }\end{array}$ & $\begin{array}{c}\text { SUBBAND } 3 \\
\text { Sig(2-tailed) }\end{array}$ & $\begin{array}{c}\text { SUBBAND } 4 \\
\text { Sig(2-tailed) }\end{array}$ & $\begin{array}{c}\text { SUBBAND } 5 \\
\text { Sig(2-tailed) }\end{array}$ \\
\hline $\begin{array}{l}\text { First Relaxation } \\
\text { Physical Stress }\end{array}$ & $\rightarrow$ & 0,056 & 0,082 & $0,014^{*}$ & $0,004^{*}$ & $0,001 *$ \\
\hline $\begin{array}{l}\text { First Relaxation } \\
\text { Cognitive Stress }\end{array}$ & $\rightarrow$ & $0,000 *$ & 0,000 & 0,000 & 0,000 & $0,000 *$ \\
\hline $\begin{array}{l}\text { First Relaxation } \\
\text { Emotional Stress }\end{array}$ & $\rightarrow$ & 0,328 & 0,343 & 0,389 & 0,328 & 0,184 \\
\hline VARIANCE & & $\begin{array}{l}\text { SUBBAND } 1 \\
\text { Sig(2-tailed) }\end{array}$ & $\begin{array}{c}\text { SUBBAND } 2 \\
\text { Sig(2-tailed) }\end{array}$ & $\begin{array}{l}\text { SUBBAND } 3 \\
\text { Sig(2-tailed) }\end{array}$ & $\begin{array}{c}\text { SUBBAND } 4 \\
\text { Sig(2-tailed) }\end{array}$ & $\begin{array}{c}\text { SUBBAND } 5 \\
\text { Sig(2-tailed) }\end{array}$ \\
\hline $\begin{array}{l}\text { First Relaxation } \\
\text { Physical Stress }\end{array}$ & $\rightarrow$ & $0,000 *$ & 0,082 & $0,014^{*}$ & $0,004 *$ & $0,001 *$ \\
\hline $\begin{array}{l}\text { First Relaxation } \\
\text { Cognitive Stress }\end{array}$ & $\rightarrow$ & $0,000 *$ & $0,001 *$ & $0,000 *$ & $0,000 *$ & $0,000 *$ \\
\hline $\begin{array}{l}\text { First Relaxation } \\
\text { Emotional Stress }\end{array}$ & $\rightarrow$ & $0,000 *$ & 0,328 & 0,389 & 0,328 & 0,184 \\
\hline
\end{tabular}

In Figure 6 there is a significant increase in the magnitude of power spectral density in the case of cognitive stress for the second EMD sub band signal.

EDA sub band signals decomposed using the EMD method were analyzed to see if there was a statistical difference in terms of energy and variance characteristics between the first relaxation and physical stress condition, first relaxation and cognitive stress condition, first relaxation and emotional stress condition. $p$ values obtained from the analysis of each EMD sub bands and each stages according

to the energy and variance features are given in the Table 2 . If $\mathrm{p}<0.05$, it can be said that there is a significant difference between the two groups. As seen from Table 2, there are significant differences between first relaxation and cognitive stress in all EMD sub bands of EDA signals in terms of energy and variance characteristics. In terms of energy, there is a significant difference in the $3^{\text {rd }}, 4^{\text {th }}$, and $5^{\text {th }}$ EMD sub bands of physical stress, but the level of difference in the cognitive stress stage is more significant than the other stages. In terms of variance, there is a significant difference in the 1st,3rd, 4th, and 5th EMD sub bands of physical stress, but the level of difference in the cognitive stress stage is more significant than the other stages. When evaluated in terms of energies, there is no statistically significant difference between first relaxation and emotional stres stages. When evaluated in terms of variances, it is seen that there is only a significant difference in subband 1 .

As seen from Table 2, there is a significant difference for the cognitive stress situation in the EDA signals, which are decomposed using EMD method.

\subsection{Classification Results}

Classification of EDA signals according to different situations has been done by using the calculated features of EMD subband signals. Variance and energy features of IMF1 and IMF2 subband signals are applied as an input to the classifiers. The input matrix $38 \times 5$ ( $38=19$ subject $\times 2$ different stage (for example: first relaxation (0), physical stress $(1)) \times(5=4$ features + group $)$ was used to classify each condition. In this paper, 10 -fold cross validation is used to train the classifiers. Classifier accuracy was calculated with equation 2 .

$$
\begin{aligned}
& \text { Accuracy }=\frac{(T P+T N)}{T P+T N+F N+F P} \\
& \text { Precision }=\frac{T P}{T P+F P} \\
& \text { Specificity }=\frac{T N}{T N+F N} \\
& \text { Sensitivity }=\frac{T P}{T P+F N} \\
& \mathrm{~F}-\text { score }=\frac{\left(1+\beta^{2}\right) \times \text { sensitivity } \times \text { precision }}{\left(\beta^{2} \times \text { sensitivity }\right)+\text { precision }}
\end{aligned}
$$

Accuracy gives information about correctly classified samples. It was calculated using (2). Sensitivity is also called Recall; it is the number of positive samples classified correctly and indicates the classifier's ability to correctly classify the positive class. Specificity indicates the measure of negative samples classified as negative. Precision is a measure of how many samples classified as positive are truly positive. It shows how much the positive classification of negative class can be avoided [30]. Evaluation of this metrics were given in (3) (4) (5). The F- $(\beta=1)$ score is a typical measure of binary classification that can be interpreted as an average of precision and sensitivity as seen (6)[30]. In the 
classification of physical stress stages for first relaxation, the highest classifier accuracy was obtained as MLP with an accuracy of $78.94 \%$. The lowest classifier accuracy was obtained from SVM classifier with $63.15 \%$. When evaluated in terms of all other performance metrics in classification of physical stress stages with first relaxation, MLP showed the highest score in all performance metrics. In the classification of cognitive stress stages with first relaxation, the highest classifier accuracy was classified as MLP with $97.36 \%$ accuracy. The lowest classifier accuracy was classified as KNN classifier with $81.57 \%$. When evaluated in terms of all other performance metrics in the classification of first relaxation and cognitive stress stages, MLP showed the highest score in all performance metrics. In the classification of emotional stress stages with first relaxation, the highest classifier accuracy was classified as KNN with an accuracy of $63.15 \%$. The lowest classifier accuracy was classified as KNN classifier with $52.63 \%$. When the classifier performances are evaluated in general the highest accuracy was obtained in the classification of first rest period cognitive stress period with all classification methods ref Table 3. Additionally, the highest accuracy was obtained in MLP method among the other two classification algorithm. Also, the lowest accuracy is seen in first rest period emotional stress period. In the classification of cognitive stress period and first rest period, the best classification success was achieved compared to other situations. Classification performance was $97.36 \%, 84.21 \%$, and 81.57 $\%$ using MLP, SVM and KNN respectively.

Table 3. Classification Results

\begin{tabular}{|c|c|c|c|}
\hline $\begin{array}{c}\text { First Relaxation } \rightarrow \\
\text { Physical Stress }\end{array}$ & $\overline{\text { SVM }}$ & $\overline{\text { KNN }}$ & MLP \\
\hline Sensitivity & 0,684211 & 0,631579 & 0,72 \\
\hline Specificity & 0,578947 & 0,684211 & 0,923077 \\
\hline Precision & 0,619048 & 0,666667 & 0,947368 \\
\hline F-score & 0,65 & 0,648649 & 0,818182 \\
\hline Accuracy & 0,631579 & 0,657895 & $\mathbf{0 , 7 8 9 4 7 4}$ \\
\hline $\begin{array}{c}\text { First Relaxation } \rightarrow \\
\text { Cognitive Stress }\end{array}$ & SVM & KNN & MLP \\
\hline Sensitivity & 0,736842 & 0,789474 & 1 \\
\hline Specificity & 0,947368 & 0,842105 & 0,95 \\
\hline Precision & 0,933333 & 0,833333 & 0,947368 \\
\hline F-score & 0,823529 & 0,810811 & 0,972973 \\
\hline Accuracy & $0,842105^{*}$ & $0,815789 *$ & $0,973684 *$ \\
\hline $\begin{array}{c}\text { FirstRelaxation } \rightarrow \\
\text { Emotional Stress }\end{array}$ & SVM & KNN & MLP \\
\hline Sensitivity & 0,526316 & 0,631579 & 0,615385 \\
\hline Specificity & 0,421053 & 0,368421 & 0,75 \\
\hline Precision & 0,47619 & 0,5 & 0,842105 \\
\hline F-score & 0,5 & 0,55814 & 0,711111 \\
\hline Accuracy & 0,526316 & 0,631579 & 0,615385 \\
\hline
\end{tabular}

In different stress situations, our body's responses to stress also differ. These stress factors can be caused by emotional factors such as excitement, sadness, or can occur when looking for a solution to a problem encountered in daily life or when doing mental calculation in an exam. Also stress is shown as one of the causes of heart diseases [31]. One of our body's first responses to these stress factors will be to secrete sweat. This secreted sweat will cause an increase in skin conductivity. This conductivity occurring in the skin is the source of different EDA signals. When evaluated from this point of view, analyzing the change in EDA signals in different stress situations allows us to evaluate the response of our body against stress. For example, Berberoğlu at al. [32] conducted a preliminary study for the wearable safety system that can help prevent occupational accidents. In this study, they said that EDA parameters can be important in determining the stress of the person.

When the studies in literature are examined, studies on time domain such as peaks, peak numbers, max points of EDA signals, and mean values of EDA signals amplitude are observed. Some researchers, such as Ghaderian and Abbasi, used time, frequency, and wavelet properties to predict mental stress using the EDA signal [33]. These studies are based on driving tasks, various images, arithmetic problems, and sound [34, 35]. Svetlak et al. [36] reported that the amplitude of the EDA signals increased significantly during the Stroop color test.

This study evaluates different types of stress using EDA signals. In our study, the detailed analysis of EDA signals showed that the change in cognitive stress was more important than the other situation $(\mathrm{p}<0.05)$. Energy and variance values for EDA signal were seen to have increased significantly in the case of cognitive stress. When the variance and energy properties of the subband signals obtained using the EMD method were evaluated, it was seen that there were significant changes in physical stress as well as cognitive stress. It was observed that EDA signals increased significantly when subjects were exposed to situations requiring mental performance. As classification results, cognitive stress period and first rest period features are classified with higher classification rate from other stages as $97.36 \%, 84.21 \%$, and $81.57 \%$ using MLP, SVM and KNN respectively.

Detection of different attention states using EDA signals can be useful for different Human Computer Interference applications. For example, in the literature, changes in EDA signals have been analyzed by using cognitive stress in determining the stress level of drivers during simulated driving conditions [36], and emotional stress factors in the assessment of consciousness in unconscious patients [37].

According to the results of this study, when individuals are exposed to emotional, physical and cognitive stress, the most change in skin conductance occurs when they are exposed to cognitive stress. In addition, in case of cognitive and physical stress, the electrodermal activity of the person increases 
significantly compared to the resting state. In case of emotional stress, there are no significant differences according to the resting state. However, in this study, the movie watched during emotional stress may have affected everyone differently. In order to provide more clear information about emotional stress, emotional stress can be evaluated by preparing a movie with different emotions inside. The results of this study show that different stress situations can be successfully analyzed using EDA signals. This study proposed an approach that uses basic signal features (energy and variance) to analyze and classify changes in different stress situations. In addition, for other users who will use the dataset used in the study, it contributes to the literature in the evaluation of EDA signals in the dataset.

In future study, the classification accuracy performance can be increased by using different decomposition methods to EDA signals or using different the features.

\section{REFERENCES}

[1].W. Boucsein, Electrodermal activity. Springer Science \& Business Media, 2012.

[2].H. ÖZBEK and D. J. S. B. D. Nazan, "Determination of the Hemispheric Difference at Attention Level in Sportsman," vol. 19, no. 2, pp. 93-101.

[3].G. R. J. Y. J. Kim, S. S. Kim, W. Y. Jang, J. H. Kim, and S. W. Baik, "Implementation of Electrodermal Activity Measurement System using Algometer and Bio_Potential Measuring System," presented at the International Conference on Chemistry, Biomedical and Environment Engineering (ICCBEE'14), Antalya (Turkey) Oct 7-8, 2014 [4].D. Nazan, "Sağlıklı Kişilerde ve Hipertiroidili Hastalarda Elektrodermal Aktivite Bulgularının İncelenmesi," Uzmanlık Tezi, Tıp Fakültesi, Fizyoloji Anabilim Dalı, Erciyes Üniversitesi, 1996.

[5].M. Tarvainen, P. Karjalainen, A. Koistinen, and M. Valkonen-Korhonen, "Principal component analysis of galvanic skin responses," in Proceedings of the 22nd Annual International Conference of the IEEE Engineering in Medicine and Biology Society (Cat. No. O0CH37143), 2000, vol. 4, pp. 3011-3014: IEEE.

[6].J. Xiong, "Design of health relaxation system based on biofeedback from finger sensors," in 2010 International Conference on Innovative Computing and Communication and 2010 Asia-Pacific Conference on Information Technology and Ocean Engineering, 2010, pp. 127-128: IEEE.

[7].W. J. B. Boucsein, EL vd, Schut, MH, Westerink, JHDM, Herk, J. v., and K. Tuinenbreijer, "Electrodermal activity: Springer Science \& Business Media," 2012.

[8].S. Zhang, S. Hu, H. H. Chao, X. Luo, O. M. Farr, and R. L. J. N. Chiang-shan, "Cerebral correlates of skin conductance responses in a cognitive task," vol. 62, no. 3, pp. 1489-1498, 2012.

[9].M. M. BRADLEY and P. J. LANG, "25 Emotion and Motivation."
[10].C. Setz, B. Arnrich, J. Schumm, R. Marca, G. Tröster, and U. Ehlert, "Discriminating stress from cognitive load using a wearable EDA device," IEEE Transactions on Information Technology in Biomedicine, vol. 14, pp. 410417, 01/01 2010.

[11].A. D. J. P. Rabavilas and Psychosomatics, "Electrodermal activity in low and high alexithymia neurotic patients," vol. 47, no. 2, pp. 101-104, 1987.

[12].J. H. Satterfield and M. E. J. P. Dawson, "Electrodermal correlates of hyperactivity in children," vol. 8, no. 2, pp. 191$197,1971$.

[13].J. A. Healey and R. W. J. I. T. o. i. t. s. Picard, "Detecting stress during real-world driving tasks using physiological sensors," vol. 6, no. 2, pp. 156-166, 2005.

[14].J. Birjandtalab, D. Cogan, M. B. Pouyan, and M. Nourani, "A non-EEG biosignals dataset for assessment and visualization of neurological status," in 2016 IEEE International Workshop on Signal Processing Systems (SiPS), 2016, pp. 110-114: IEEE.

[15].A. L. Goldberger et al., "PhysioBank, PhysioToolkit, and PhysioNet: components of a new research resource for complex physiologic signals," vol. 101, no. 23, pp. e215e220, 2000.

[16]. E. Huang et al., "The empirical mode decomposition and the Hilbert spectrum for nonlinear and non-stationary time series analysis," vol. 454, no. 1971, pp. 903-995, 1998. [17].K. Drakakis, "Empirical mode decomposition of financial data," in International Mathematical Forum, 2008, vol. 3, no. 25, pp. 1191-1202: Citeseer.

[18].Z.-E. H. Slimane and A. J. D. S. P. Naït-Ali, "QRS complex detection using Empirical Mode Decomposition," vol. 20, no. 4, pp. 1221-1228, 2010.

[19].M. Blanco-Velasco, B. Weng, K. E. J. C. i. b. Barner, and medicine, "ECG signal denoising and baseline wander correction based on the empirical mode decomposition," vol. 38, no. 1, pp. 1-13, 2008.

[20].R. B. Pachori, V. J. C. m. Bajaj, and p. i. biomedicine, "Analysis of normal and epileptic seizure EEG signals using empirical mode decomposition," vol. 104, no. 3, pp. 373381, 2011.

[21].D.-x. Zhang, X.-p. Wu, and X.-j. Guo, "The EEG signal preprocessing based on empirical mode decomposition," in 2008 2nd International Conference on Bioinformatics and Biomedical Engineering, 2008, pp. 2131-2134: IEEE.

[22].A. Gautam, N. Simoes-Capela, G. Schiavone, A. Acharyya, W. De Raedt, and C. Van Hoof, "A Data Driven Empirical Iterative Algorithm for GSR Signal PreProcessing," in 2018 26th European Signal Processing Conference (EUSIPCO), 2018, pp. 1162-1166: IEEE.

[23].N. E. Huang, Z. Shen, and S. R. J. A. r. o. f. m. Long, "A new view of nonlinear water waves: the Hilbert spectrum," vol. 31, no. 1, pp. 417-457, 1999.

[24].N. E. Huang et al., "A confidence limit for the empirical mode decomposition and Hilbert spectral analysis," vol. 459, no. 2037, pp. 2317-2345, 2003.

[25].G. Rilling, P. Flandrin, and P. Goncalves, "On empirical mode decomposition and its algorithms."

[26].H. Erkaymaz, M. Ozer, and İ. M. Orak, "Detection of directional eye movements based on the electrooculogram 
signals through an artificial neural network," Chaos, Solitons \& Fractals, vol. 77, pp. 225-229, 2015/08/01/ 2015.

[27].C. C. Aggarwal, "Neural networks and deep learning." [28].URL: h. t. c. k.-k.-n. neighbors-1-a4707b24bd1d. (Access date : 19.07.2019).

[29].C. Cortes and V. J. M. 1. Vapnik, "Support-vector networks," vol. 20, no. 3, pp. 273-297, 1995.

[30].A. Gosain and S. Sardana, "Handling class imbalance problem using oversampling techniques: A review," in 2017 International Conference on Advances in Computing, Communications and Informatics (ICACCI), 2017, pp. 7985: IEEE.

[31].S. U. Amin, K. Agarwal, and R. Beg, "Genetic neural network based data mining in prediction of heart disease using risk factors," in 2013 IEEE Conference on Information \& Communication Technologies, 2013, pp. 1227-1231: IEEE.

[32].E. Beberoglu, M. Tokmakçi, and A. Ozdemir, Is kazalarini onleyebilmek icin bakim/onarim personelinin kullanabilecegi bir giyilebilir emniyet sisteminin tasarlanmasi. 2019.
[33].P. Ghaderyan and A. J. I. J. o. P. Abbasi, "An efficient automatic workload estimation method based on electrodermal activity using pattern classifier combinations," vol. 110, pp. 91-101, 2016.

[34].J. Kim, E. J. I. t. o. p. a. André, and m. intelligence, "Emotion recognition based on physiological changes in music listening," vol. 30, no. 12, pp. 2067-2083, 2008.

[35].W. Wen, G. Liu, N. Cheng, J. Wei, P. Shangguan, and W. J. I. T. o. A. C. Huang, "Emotion recognition based on multi-variant correlation of physiological signals," vol. 5, no. 2, pp. 126-140, 2014.

[36].J. S. K. Ooi, S. A. Ahmad, Y. Z. Chong, S. H. M. Ali, G. Ai, and H. Wagatsuma, "Driver emotion recognition framework based on electrodermal activity measurements during simulated driving conditions," in 2016 IEEE EMBS Conference on Biomedical Engineering and Sciences (IECBES), 2016, pp. 365-369: IEEE.

[37].J. Luauté et al., "Electrodermal reactivity to emotional stimuli in healthy subjects and patients with disorders of consciousness," vol. 61, no. 6, pp. 401-406, 2018. 